\title{
Estimativa do Balanço Térmico por Radiação em Vacas Holandesas Expostas ao Sol e à Sombra em Ambiente Tropical
}

\author{
Roberto Gomes da Silva ${ }^{1}$
}

\begin{abstract}
RESUMO - É apresentado um método para a estimativa do balanço térmico por radiação em vacas da raça Holandesa expostas ao sol e sob a sombra em uma pastagem, com o objetivo de estabelecer um padrão morfológico mais adequado para esses animais em região tropical. O método envolve a determinação das características do pelame (área de superfície de malhas negras, espessura da capa, comprimento e diâmetro dos pêlos, número de pêlos por unidade de área), temperatura da superfície da capa e da epiderme e variáveis ambientais (irradiância solar, temperatura do ar e de globo, vento). Foi determinada a quantidade de radiação de ondas curtas efetivamente transmitida através do pelame e que atinge as camadas profundas da epiderme. Demonstrou-se, por intermédio de um exemplo prático, que as áreas de pelame negro apresentaram balanço radiante mais elevado que as áreas brancas, mas estas últimas possuiam maior transmissividade para a radiação de ondas curtas. Animais predominantemente negros foram melhor protegidos contra a radiação de ondas curtas que os predominantemente brancos, especialmente quando a capa de pelame é pouco espessa (até 4 a $7 \mathrm{~mm}$ ), mais adequada para ambientes tropicais.
\end{abstract}

Palavras-chave: balanço térmico, pelame, radiação, vacas Holandesas

\section{Estimate of Radiation Heat Balance of Holstein Cows in the Sun and under the Shade in a Tropical Environment}

\begin{abstract}
A method is presented to estimate the radiation heat balance of Holstein cows exposed to sun and under shade of a pasture, to contribute with a morphological characteristics better adequate for these animals in a tropical environment. The methods involved the haircoat characteristics (surface area of black spots, coat thickness, hair length, hair diameter, number of hairs per unit of area), skin and coat surface temperatures and environmental variables (solar irradiance, wind speed, air and globe temperatures). The amount of shortwave energy which effectively is transmitted through the haircoat and that reach deply the epiderms layers was obtained. It was demonstrated by a practical example that the black areas of the coat presented higher radiation heat balance than that of the white areas, but those had greater transmissivity for shortwave radiation. Predominantly black cows were better protected against shortwave radiation than the white ones, especially if the animals presented thin (4 to $7 \mathrm{~mm}$ or less) haircoats, the most suitable for tropical environments.
\end{abstract}

Key Words: haircoat, Holstein cows, radiation balance

\section{Introdução}

A termorregulação nos animais tem sido extensivamente estudada em câmaras climáticas e calorímetros, nos quais as condições ambientes podem ser facilmente controladas. Entretanto, o ambiente externo, no qual os animais vivem normalmente, é muito mais complexo, pois fatores como velocidade do vento, radiação, temperatura e umidade do ar modificam-se continuamente e alterações em uma ou outra dessas variáveis podem causar mudanças significativas em todos os componentes do balanço térmico.

Diversos trabalhos têm sido publicados a respeito das relações entre o organismo animal e o ambiente radiante (BAKKEN, 1976; CAMPBELL, 1977;
McARTHUR, 1987; MONTEITH, 1974; MONTEITH e UNSWORTH, 1991; e STAFFORD-SMITH et al., 1985). Não há, entretanto, referências específicas ao balanço térmico radiante de vacas leiteiras expostas em campo aberto a um ambiente tropical.

Existem diferenças marcadas entre o ambiente térmico de vacas pastando em uma região tropical e o encontrado por elas em regiões temperadas; nestas, a temperatura do ar e a pressão de vapor encontramse geralmente abaixo dos valores equivalentes do corpo de um animal, o qual então pode facilmente eliminar energia térmica para o ambiente através de convecção, evaporação e radiação. Por outro lado, em regiões tropicais, a temperatura do ar frequentemente excede a temperatura corporal e a termólise 
por convecção é prejudicada. Se a região for úmida, elevados níveis de pressão de vapor do ar podem dificultar seriamente a evaporação cutânea e a respiratória, causando, assim, severo estresse de calor.

Em adição, a temperatura radiante das vizinhanças do animal pode ser próxima ou maior que a temperatura da superfície corporal, visto que a quantidade de energia térmica recebida por radiação é maior que a eliminada. É inquestionável que nas regiões tropicais as trocas térmicas por radiação entre animais e ambiente assumem grande importância, pois determinam em muitos casos a diferença entre um ambiente confortável e outro intolerável.

A importância da pigmentação e de outras características do pelame para a troca térmica radiante nos animais tem sido objeto de considerável discussão (CENA e MONTEITH, 1975; COWLES, 1967; DAWSON e BROWN, 1970; FINCH et al., 1984; HAMILTON, 1973; HAMILTON e HEPPNER, 1967; HUTCHINSON e BROWN, 1969; KOVARIK, 1973; McARTHUR, 1987; SILVA et al., 1988; WALSBERG et al., 1978; e GEBREMEDHIN et al., 1997). É geralmente aceito que os animais com pelame escuro - e, portanto, maior absorção da radiação térmica - são mais sujeitos ao estresse de calor que os de pelame claro.

Entretanto, tem sido demonstrado que pelames claros apresentam maior penetração da radiação solar que os escuros (CENA e MONTEITH, 1975; HUTCHINSON e BROWN, 1969; e WALSBERG et al., 1978), e a transmissão dessa radiação ocorre através da capa dependente das propriedades estruturais e físicas da mesma (espessura da capa, comprimento dos pêlos, pigmentação dos pêlos, número de pêlos por unidade de área e diâmetro dos pêlos). Constam da Tabela 1 algumas medidas destas características em bovinos da raça Holandesa, com destaque para as realizadas em ambiente tropical. No que se refere aos efeitos da radiação de ondas curtas, particularmente a ultravioleta, a transmissão através do pelame apresenta importância fundamental e depende ainda da pigmentação da epiderme (SILVA et al., 1988).

Os propósitos do presente trabalho foram, em primeiro lugar, apresentar um modelo operacional para a estimação da troca térmica radiante em vacas da raça Holandesa mantidas em ambiente tropical e, em segundo lugar, verificar a melhor caracterização morfológica para os animais nesse ambiente.

\section{Material e Métodos}

\section{Modelo}

A manutenção de temperatura interna relativamente constante em um animal homeotérmico depende do balanço entre a energia térmica originada pelos processos metabólicos (M), das trocas térmicas por radiação entre o organismo e o ambiente (R), e as perdas térmicas por convecção (C), evaporação (E) e condução (K). As trocas por condução geralmente são desprezíveis, exceto quando os animais se acham deitados:

$$
\mathbf{M} \pm \mathbf{R}-\mathbf{C}-\mathbf{E}=\mathbf{0}
$$

Um bovino em pé em campo aberto recebe energia térmica radiante basicamente das seguintes fontes: (1) radiação solar direta de ondas curtas, (2) radiação celeste difusa de ondas curtas, (3) radiação de ondas curtas refletida pelo solo, vegetação e objetos vizinhos, (4) radiação de ondas longas procedente da atmosfera e (5) radiação de ondas longas emitidas pelo solo e superfícies vizinhas. A radiação total de ondas curtas é representada por St e a de ondas longas, por $\mathrm{L}$, de modo que se pode então definir:

$$
\mathbf{R}=\mathbf{S}_{\mathbf{t}}+\mathbf{L}
$$

Entretanto, a quantidade de ondas curtas efetivamente absorvidas pelo animal depende da refletividade da sua superfície externa com relação a essas ondas. Considerando-se que a refletividade é na realidade uma função das características não apenas do pelame, como também da epiderme, pode-se definir a refletividade efetiva como a proporção da energia radiante incidente, que é refletida pela superfície em decorrência da associação das características físicas e morfológicas do pelame e da epiderme. Segundo CENA e MONTEITH (1975), a refletividade efetiva pode ser estimada como:

$$
\rho *=\frac{\left[\rho_{\mathrm{s}}\left(1-\rho_{\mathrm{e}}\right)-\alpha_{\mathrm{s}} \rho_{\mathrm{e}}\right] \operatorname{senh} x+\kappa \rho_{\mathrm{e}} \cosh x}{\left[\rho_{\mathrm{s}}\left(1-\rho_{\mathrm{e}}\right)+\alpha_{\mathrm{s}}\right] \operatorname{senh} x+\kappa \cosh x}
$$

sendo

$$
\begin{aligned}
& \quad \kappa=\left(\alpha_{\mathrm{s}}^{2}+2 \rho_{\mathrm{s}} \alpha_{\mathrm{s}}\right)^{1 / 2} \\
& \mathrm{x}=\mathrm{kp}^{\mathrm{E}}=\operatorname{nd}\{\tan [\arccos (\mathrm{E} / \mathrm{C})]\}
\end{aligned}
$$

em que n é a densidade numérica do pelame; d, o diâmetro médio dos pêlos; $\mathrm{E}$, a espessura da capa de pelame; $\mathrm{C}$, o comprimento médio dos pêlos; $\rho$ e, a refletividade da epiderme; $\rho$ s, a refletividade da superfície do pelame; $\rho$, a absorvidade da superfície do 
Rev. bras. zootec.

pelame; e senh $x$ e $\cosh x$, o seno hiperbólico e o cosseno hiperbólico de $x$, respectivamente.

$$
\begin{aligned}
& \operatorname{senh} x=0,5\left(\mathrm{e}^{\mathrm{x}}-\mathrm{e}^{-\mathrm{x}}\right) \\
& \cosh x=0,5\left(\mathrm{e}^{\mathrm{x}}+\mathrm{e}^{-\mathrm{x}}\right)
\end{aligned}
$$

Por outro lado, a transmissividade efetiva do pelame para a radiação de ondas curtas é dada por:

$$
\tau *=\frac{\kappa}{\left[\rho_{\mathrm{s}}\left(1-\rho_{\mathrm{e}}\right)+\alpha_{\mathrm{s}}\right] \operatorname{senh} x+\kappa \cosh x} \ldots
$$

e a absorvidade efetiva por:

$$
\alpha^{*}=1-\rho^{*}-\tau^{*}\left(1-\rho_{\mathrm{e}}\right)
$$

Entretanto, pode-se verificar que o valor $x$ aumenta diretamente com o ângulo dos pêlos em relação à superfície da epiderme. Em animais mantidos em ambiente tropical, a tendência é reduzir esse ângulo, de modo a aumentar a condutividade térmica da capa e eliminar calor corporal mais facilmente. Quando $x>2,7$, a diferença entre $\operatorname{senh} x$ e $\cosh x$ é muito pequena; aumentando o valor de $x$ acima desse limite, essa diferença tende a zero e, nestas condições, pode-se simplificar as equações acima:

$$
\begin{aligned}
& \rho *=\frac{\rho_{\mathrm{s}}\left(1-\rho_{\mathrm{e}}\right)-\alpha_{\mathrm{s}} \rho_{\mathrm{e}}+\kappa \rho_{\mathrm{e}}}{\rho_{\mathrm{s}}\left(1-\rho_{\mathrm{e}}\right)+\alpha_{\mathrm{s}}+\kappa} \\
& \tau *=\frac{\kappa}{\rho_{\mathrm{s}}\left(1-\rho_{\mathrm{e}}\right)+\alpha_{\mathrm{s}}+\kappa}
\end{aligned}
$$

Sabe-se, todavia, que as vacas Holandesas são malhadas, com áreas de pelame negro e áreas brancas, cada uma com diferente valor de absorvidade para a radiação de ondas curtas, o que resulta em diferentes temperaturas. Se $n$ for a proporção da área total de superfície corporal, que é composta por pelame negro, e $b$, a proporção da área que corresponde a pelame branco, a quantidade de radiação de ondas curtas absorvida e transmitida pelo pelame e pela epiderme de um animal seria então dada por $\mathrm{S}_{\mathrm{t}}\left[n\left(1-\rho_{\mathrm{n}}{ }^{*}\right)+b\left(1-\rho_{\mathrm{b}}{ }^{*}\right)\right]$, em que $\boldsymbol{\rho}_{\mathbf{n}} *$ e $\boldsymbol{\rho}_{\mathbf{b}} *$ são os coeficientes de refletividade efetiva das malhas negras e brancas, respectivamente.

\section{Radiação de ondas curtas}

A irradiância da superfície terrestre, assumindo-se uma atmosfera totalmente limpa de aerossóis, é $S_{p}=1372,7$ $\cos \theta$, em que $\theta$ éoângulo zenital do sol ou ângulo poreste formado com relação ao zênite e dado por:

$$
\begin{aligned}
& \cos \delta=(\cos L)(\cos \theta)(\cos H)+(\operatorname{sen} L)(\operatorname{sen} \delta) \\
& \text { em que } \\
& L=\text { latitude do local (graus), valor negativo para }
\end{aligned}
$$
o hemisfério sul;

$\delta=$ declinação do sol (graus) $=23,45 \operatorname{sen}[(360 /$ $365)(284+d)]$ $\mathrm{d}=$ número ordinal do dia do ano (1 a 365);

$\mathrm{H}=$ ângulo horário do sol (graus) $=15(\mathrm{~h}-12)$; e

$\mathrm{h}=$ hora do dia (0 a 24, em valor decimal).

Considerando que a atmosfera apresenta quantidades variáveis de aerossóis (vapores e partículas sólidas em suspensão), aplica-se a lei de Beer e obtém-se a irradiância como:

$$
\mathrm{S}=\mathrm{S}_{\mathrm{p}} \mathrm{e}^{-\mathrm{mt}}
$$

em que $m=\sec \theta=1 /(\cos \theta)$ é a massa atmosférica atravessada pela radiação e $t$, o coeficiente de turbidez. De acordo com MONTEITH e UNSWORTH (1991), o valor de t pode variar entre 0,07 (atmosfera muito clara e límpida, como a encontrada no alto de montanhas) e 0,6 (atmosfera altamente poluída, como em cidades industriais). Nas condições usuais do campo, a estimativa $t=0,1$ é perfeitamente aceitável.

Assumindo que, em geral, apenas $4,4 \%$ da radiação de ondas curtas incidente em um dia claro poderiam ser considerados como radiação difusa (McCULLOUGH e PORTER 1971) e considerando a ocorrência de nuvens, STAFFORD-SMITH et al. (1985) propuseram que a incidência de ondas curtas sobre uma superfície plana horizontal fosse dividida em radiação direta $\left(\mathrm{S}_{\text {dir }}\right)$, difusa $\left(\mathrm{S}_{\text {dif }}\right)$ e refletida no solo ao redor do animal $\left(\mathrm{S}_{\text {ref }}\right)$ :

$$
\begin{gathered}
\mathbf{S}_{\text {dir }}=0,956(1-\mathrm{c}) \mathbf{S} \\
\mathbf{S}_{\text {dif }}=0,044 \mathrm{~S}+0,956 \mathrm{cS} \\
\mathbf{S}_{\text {ref }}=\rho_{\mathrm{g}} \mathbf{S}
\end{gathered}
$$

em que c é a proporção do céu coberta de nuvens e $\rho_{\mathrm{g}}$, a refletividade da superfície do solo. Em adição, cerca de $40 \%$ da radiação difusa e da refletida alcançariam um animal sob a sombra de uma árvore.

As equações acima supõem que a superfície receptora seja plana e horizontal. Entretanto, o corpo de uma vaca não é uma superfície plana, assemelhando-se antes a um cilindro horizontal de comprimento $h$ e raio $r$. Para se estimar a quantidade de radiação interceptada, a irradiância total deveria ser multiplicada por um fator de conformação, que para um cilindro horizontalé

$$
\begin{gathered}
F_{c}=\frac{2 r h(1 / \operatorname{sen} \beta)\left[1-\left(\cos ^{2} \beta\right)\left(\cos ^{2} \omega\right)\right]^{1 / 2}+\pi r^{2}(1 / \tan \beta)(\cos \omega)}{2 \pi r h+2 \pi r^{2}} \\
\ldots[8]
\end{gathered}
$$

conforme MONTEITH e UNSWORTH (1991), em que $\beta$ é o ângulo de elevação do sol sobre o horizonte $(90-\theta)$ e $\omega$, a orientação azimutal do eixo do cilindro com respeito ao sol. CLAPPERTON et al. (1965) determinaram com bons resultados a área de sombra projetada por ovinos adultos, comparando-a 
com a área de cilindros horizontais de dimensões equivalentes.

Considerando então o fator de conformação, o total de radiação de ondas curtas que efetivamente incide sobre um animal será dado por:

$\mathbf{S}_{\mathrm{t}}=(1-0,75 \mathrm{c})\left[(1-\mathrm{s}) \mathrm{F}_{\mathrm{c}} \mathbf{S}_{\mathrm{dir}}+(1-0,6 \mathrm{~s})\left(\mathbf{S}_{\mathrm{dif}}+\mathbf{S}_{\mathrm{ref}}\right)\right]$ ...[9]

em que $\mathrm{s}=0$ quando o animal está ao sol e $\mathrm{s}=1$, à sombra. O valor 0,75 indica que cerca de $25 \%$ da radiação solar são transmitidos através da camada de nuvens (STAFFORD-SMITH et al., 1985).

Trocas térmicas por radiação de ondas longas

Seja $\mathrm{T}_{\mathrm{RM}}$ a temperatura radiante média do ambiente que circunda o animal, estimada através de um globo negro de Vernon colocado à mesma altura do solo que o centro do tronco do corpo e protegido da radiação solar direta por um pequeno parassol (de cartão ou isopor). Se TG for a temperatura do globo, assumindo o valor da emissividade da sua superfície como 0,95, ter-se-á:

$$
\mathrm{T}_{\mathrm{RM}}=\left[(0,95 \sigma)^{-1} \mathrm{~h}_{\mathrm{C}}\left(\mathrm{T}_{\mathrm{G}}-\mathrm{T}_{\mathrm{a}}\right)+\mathrm{T}_{\mathrm{G}}^{4}\right]^{1 / 4},{ }^{\mathrm{o}} \mathrm{K}
$$

em que $\mathrm{h}_{\mathrm{C}}$ é o coeficiente de convecção do globo e $\sigma=5,6697 \times 10^{-8} \mathrm{~W} /\left(\mathrm{m}^{2}{ }^{\circ} \mathrm{K}\right)$, a constante de StefanBoltzmann. $\mathrm{O}$ valor de $\mathrm{h}_{\mathrm{C}}$ dependerá da ocorrência de convecção natural ou convecção forçada:

Convecção forçada: $h_{C}=0,38 \mathrm{kd}^{-1} \operatorname{Re}^{0,6} \operatorname{Pr}^{1 / 3} \ldots[11]$

Convecção natural:

$$
\mathrm{h}_{\mathrm{C}}=\mathrm{kd}^{-1}\left(2+0,43 \mathrm{Gr}^{1 / 4} \operatorname{Pr}^{1 / 4}\right) \ldots[12]
$$

em que

$\mathrm{Re}=$ número de Reynolds $=\mathrm{Vd} v^{-1}$;

$\mathrm{Gr}=$ número de Grashof $=\mathrm{gd}^{3} v^{-2} \mathrm{~T}_{\mathrm{a}}{ }^{-1}\left(\mathrm{~T}_{\mathrm{G}}-\mathrm{T}_{\mathrm{a}}\right) ; \mathrm{e}$

$\operatorname{Pr}=$ número de Prandtl $=\rho c_{\mathrm{P}} v \mathrm{k}^{-1}$.

V é a velocidade do vento $(\mathrm{m} / \mathrm{s}) ; \mathrm{d}$, o diâmetro do globo $(\mathrm{m})$; Ta, a temperatura do ar $\left({ }^{\circ} \mathrm{K}\right) ; \mathrm{T}_{\mathrm{G}}$, a temperatura do globo $\left({ }^{\circ} \mathrm{K}\right) ; \mathrm{k}$, a condutividade térmica do ar na temperatura $\mathrm{T}_{\mathrm{a}}\left[\mathrm{W} /\left(\mathrm{m} .{ }^{\circ} \mathrm{C}\right)\right] ; \rho$, a densidade do ar na temperatura $\mathrm{T}_{\mathrm{a}}(\mathrm{kg} / \mathrm{m} 3) ; v$, viscosidade cinemática do ar à temperatura $\mathrm{T}_{\mathrm{a}}\left(\mathrm{m}^{2} / \mathrm{s}\right) ; \mathrm{c}_{\mathrm{P}}, \mathrm{o}$ calor específico do ar na temperatura $\mathrm{T}_{\mathrm{a}}\left[\mathrm{J} /\left(\mathrm{kg} .{ }^{\circ} \mathrm{C}\right)\right]$; e g, a aceleração gravitacional (valor médio de $9,783 \mathrm{~m} / \mathrm{s}^{2}$ para latitudes entre $0^{\circ}$ e $23^{\circ}$ ).

Para estabelecer o tipo de convecção que está ocorrendo, comparam-se os valores Gr e Re, conforme CHAPMAN (1987), como se segue: se $\mathrm{Gr} / \mathrm{Re}^{2} \leq 0,08$, a convecção é forçada; se $\mathrm{Gr} / \mathrm{Re}^{2}>3$ a convecção é natural; se o valor for intermediário entre ambos esses limites, deve-se calcular $\mathrm{h}_{\mathrm{C}}$ para os dois casos e escolher o maior valor (MONTEITH e UNSWORTH 1991).
Uma vez que parte da radiação recebida pelo globo é de ondas curtas (difusa e refletida), a carga térmica no mesmo deve ser corrigida para esta radiação, de modo a se obter uma estimativa da radiação incidente de ondas longas. Assim, calcula-se:

$\mathrm{T}_{\mathrm{RM}}^{*}=\left[\mathrm{T}_{\mathrm{RM}}^{4}-(0,4 / \sigma)\left(\mathbf{S}_{\mathrm{dif}}+\mathbf{S}_{\text {ref }}\right)\right]^{1 / 4},{ }^{\mathrm{o}} \mathrm{K} \ldots[13]$

Conseqüentemente, a troca térmica por radiação de ondas longas entre um animal e o ambiente será dada por $\mathrm{L}=a_{c}{ }_{c}\left(\mathrm{~T}_{\mathrm{RM}}^{* 4}-\mathrm{T}_{\mathrm{c}}^{4}\right)$, considerando que o conjunto de superfícies do ambiente, cuja temperatura média é $\mathrm{T}_{\mathrm{RM}}$, constitui um corpo negro $(\varepsilon=1)$. Porém, as áreas negras e brancas do corpo de uma vaca Holandesa apresentam diferentes temperaturas e, finalmente, ter-se-á,

$$
\mathbf{L}=\varepsilon_{\mathrm{c}} \sigma\left(\mathrm{T}_{\mathrm{RM}}^{* 4}-n \mathrm{~T}_{\mathrm{cn}}^{4}-b \mathrm{~T}_{\mathrm{cb}}^{4}\right), \mathrm{W} / \mathrm{m}^{2}
$$

em que $\varepsilon_{c}$ é a emissividade da superfície do pelame; ${ }_{\mathrm{n}} \mathrm{e}_{\mathrm{b}}$ são as proporções da área de superfície cobertas por pelame negro e branco, respectivamente; e $\mathrm{T}_{\mathrm{cn}}$ e $\mathrm{T}_{\mathrm{cb}}$ são as temperaturas das superfícies do pelame negro e branco, respectivamente.

Então, o balanço de energia radiante para um animal em campo aberto será:

$$
\mathrm{R}=\mathrm{St}\left[\boldsymbol{n}\left(\mathbf{1}-\boldsymbol{\rho}_{\mathbf{n}}^{*}\right)+{ }_{b}\left(\mathbf{1}-\boldsymbol{\rho}_{\mathbf{b}}{ }^{*}\right)\right]+\mathrm{L}, \mathrm{W} / \mathrm{m}^{2} \text {. }
$$

\section{Resultados e Discussão}

Suponha-se uma vaca Holandesa pastando ao sol às 14 h do dia 20 de outubro, em um local a $20^{\circ} 15^{\prime}$ latitude sul, em que a temperatura do ar é $27,4^{\circ} \mathrm{C}$, a velocidade do vento, $0,75 \mathrm{~m} / \mathrm{s}$, e o céu apresenta $10 \%$ de nuvens, com atmosfera límpida. O animal está colocado de lado em ângulo reto com o sol, apresentando $75 \%$ de malhas negras e as seguintes dimensões corporais: comprimento, $1,8 \mathrm{~m}$; diâmetro médio, $0,8 \mathrm{~m}$. A temperatura da superfície do pelame foi tomada com um termômetro de infravermelho Horiba modelo IT-330, o qual permite variar os valores de emissividade. As características do pelame (comprimento dos pêlos, densidade numérica e espessura da capa) foram diretamente determinadas nos animais, usando os métodos descritos por SILVA et al.(1988). As áreas de malhas negras em relação às brancas foram estimadas fotografando-se cada animal de ambos os lados, junto com padrão métrico, determinando-se a área de malhas com um planímetro. As características do pelame estão na Tabela 2. Um globo negro de $0,15 \mathrm{~m}$ de diâmetro foi colocado no mesmo local que o animal, à altura de $0,8 \mathrm{~m}$ acima do solo, e coberto por um anteparo circular de $0,25 \mathrm{~m}$ de 
Rev. bras. zootec.

Tabela 1 - Características da superfície externa de bovinos da raça Holandesa, conforme a literatura Table 1 - Outer surface characteristics of the body of a Holstein cow, after several authors

\begin{tabular}{|c|c|c|c|c|}
\hline $\begin{array}{l}\text { Característica } \\
\text { Trait }\end{array}$ & $\begin{array}{l}\text { Negro } \\
\text { Black }\end{array}$ & $\begin{array}{c}\text { Branco } \\
\text { White }\end{array}$ & $\begin{array}{l}\text { Média } \\
\text { Average }\end{array}$ & $\begin{array}{l}\text { Autor } \\
\text { Author }\end{array}$ \\
\hline Absorvidade do pelame & 0,91 & 0,45 & - & HUTCHINSONe \\
\hline \multirow[t]{2}{*}{ Hair coat absorptivity } & & & & BROWN(1969) \\
\hline & 0,91 & 0,42 & - & STEWART (1953) \\
\hline Refletividade do pelame & 0,10 & 0,42 & - & CENA(1966) \\
\hline Hair coat reflectivity & 0,09 & 0,58 & - & MOUNT(1968) \\
\hline Refletividade da epiderme & $0,18^{*}$ & $0,35^{*}$ & - & BLAXTER (1989) \\
\hline \multirow[t]{2}{*}{ Skin reflectivity } & $0,20 *$ & $0,93 *$ & - & $\begin{array}{l}\text { MONTEITHe } \\
\text { UNSWORTH(1991) }\end{array}$ \\
\hline & $0,26 * *$ & $0,39 * *$ & - & \\
\hline Diâmetro dos pêlos $(\mu \mathrm{m})$ & 43,5 & 38,4 & - & UDO(1978) \\
\hline $\begin{array}{l}\text { Hair diameter } \\
\text { Comprimento dos pêlos }(\mu \mathrm{m})\end{array}$ & - & - & 38,0 & BIANCA (1974) \\
\hline \multirow[t]{3}{*}{ Hair length } & 24,8 & $\begin{array}{c}25,6 \\
-\end{array}$ & $\begin{array}{c}- \\
13,8\end{array}$ & $\begin{array}{l}\text { UDO (1978) } \\
\text { PINHEIRO(1996) }\end{array}$ \\
\hline & - & - & 10,6 & BIANCA (1974) \\
\hline & - & - & 7,9 & ALMEIDA(1986) \\
\hline Espessura da capa $(\mu \mathrm{m})$ & - & - & 3,71 & PINHEIRO(1996) \\
\hline Coat thickness & - & - & $\begin{array}{l}2,1 \\
2,3\end{array}$ & $\begin{array}{l}\text { ALMEIDA (1986) } \\
\text { BERMANe } \\
\text { VOLCANI(1961) }\end{array}$ \\
\hline Número de pêlos $\left(\mathrm{cm}^{-1}\right)$ & 1465 & 1430 & - & $\operatorname{UDO}(1978)$ \\
\hline Number of hairs & - & - & 1435 & BIANCA (1974) \\
\hline $\begin{array}{l}\text { Proporção de área de malhas (\%) } \\
\text { Spot area }\end{array}$ & 62,4 & 37,6 & - & PEGLERJR.(1986) \\
\hline
\end{tabular}

Tabela 2 - Valores considerados para o animal usado no teste, conforme a cor das malhas Table 2 - Trait values for the animal used in the test, according to the spot colour

\begin{tabular}{|c|c|c|}
\hline Característica & Negra & Branca \\
\hline Trait & Black & White \\
\hline Espessura da capa (m) & $3,0 \times 10^{-3}$ & $3,7 \times 10^{-3}$ \\
\hline $\begin{array}{l}\text { Haircoat thickness } \\
\text { Comprimento dos pêlos (m) }\end{array}$ & $12,5 \times 10^{-3}$ & $13,8 \times 10^{-3}$ \\
\hline $\begin{array}{l}\text { Hair length } \\
\text { Diâmetro dos pêlos (m) }\end{array}$ & $43,5 \times 10^{-6}$ & $38,4 \times 10^{-6}$ \\
\hline $\begin{array}{l}\text { Hair diameter } \\
\text { Número de pêlos }\left(\mathrm{m}^{-2}\right)\end{array}$ & $1,46 \times 10^{7}$ & $1,4 \times 10^{7}$ \\
\hline $\begin{array}{l}\text { Number of hairs per unit area } \\
\text { Absorvidade da superfície do pelame }\end{array}$ & 0,91 & 0,45 \\
\hline $\begin{array}{l}\text { Haircoat surface absorptivity } \\
\text { Refletividade da superfície do pelame } \\
\text { Haircoat surface reflectivity }\end{array}$ & 0,09 & 0,42 \\
\hline $\begin{array}{l}\text { Refletividade da superfície da epiderme } \\
\text { Skin surface reflectivity }\end{array}$ & 0,18 & 0,35 \\
\hline $\begin{array}{l}\text { Transmissividade da epiderme }(0,2-0,4 \mu \mathrm{m}) \\
\text { Skin transmissivity } \\
\text { Temperatura da superfície do pelame }\left({ }^{\circ} \mathrm{C}\right)\end{array}$ & 0,098 & 0,25 \\
\hline $\begin{array}{l}\text { Haircoat surface temperature } \\
\text { Ao sol }\end{array}$ & 41,0 & 35,0 \\
\hline $\begin{array}{l}\text { Sun } \\
\text { À sombra } \\
\text { Shade }\end{array}$ & 34,0 & 32,1 \\
\hline
\end{tabular}


diâmetro para protegê-lo da radiação solar direta; o anteparo permanecia suspenso à altura de $0,5 \mathrm{~m}$ do globo, para não influir no desempenho do mesmo, e a temperatura do globo foi $38,7^{\circ} \mathrm{C}$ ao sol e $30,8^{\circ} \mathrm{C}$ à sombra. A velocidade do vento e a temperatura do ar foram tomadas simultaneamente junto ao globo por meio de um anemômetro-termômetro SPER Scientific modelo 840030.

Utilizando a equação [6], calculou-se o ângulo zenital do sol para o local, dia e hora indicados, obtendo $\theta=30^{\circ} 07^{\prime} 47^{\prime \prime}$. Assumindo $\tau=0,1$ e sendo $\beta=90-\theta=59^{\circ} 52^{\prime} 13$ ", obtém-se:

$$
\begin{gathered}
\mathrm{S}=1057,6 \mathrm{~W} \cdot \mathrm{m}^{-2} \\
\mathrm{~S}_{\text {dir }}=0,956(1-0,1)(1057,6)=909,96 \mathrm{~W} \cdot \mathrm{m}^{-2} \\
\mathrm{~S}_{\text {dif }}=0,044(1057,6)+0,956(0,1)(1057,6)=
\end{gathered}
$$
$147,64 \mathrm{~W} . \mathrm{m}^{-2}$

$$
\mathrm{S}_{\text {ref }}=0,33(1057,6)=349,01 \mathrm{~W} \cdot \mathrm{m}^{-2}
$$

$\mathrm{O}$ fator de conformação calculado pela fórmula [8] é $\mathrm{F}_{\mathrm{c}}=0,30112$. Supondo agora que o animal esteja ao sol, então s=0 e daí, aplicando a equação [9], ter-se-á:

$$
\mathbf{S}_{\mathrm{t}}=[1-0,75(0,1)][(1 * 0)(0,30112)(909,96)
$$
$(1-0)(349,01)+147,64)]=712,86 \mathrm{~W} \cdot \mathrm{m}^{-2}$

e se o animal estiver à sombra, $s=1$ e, então, $\mathrm{S}_{\mathrm{t}}=265,7 \mathrm{~W} \cdot \mathrm{m}^{-2}$.

Para a determinação da $\mathrm{T}_{\mathrm{RM}}$, obteve-se $\operatorname{Re}=7133,8, \operatorname{Pr}=0,715712$ e Gr=4.991.650,3. Uma vez que $\mathrm{Gr} / \mathrm{Re}^{2}=0,098$, calculou-se $\mathrm{h}_{\mathrm{C}}$ para os dois tipos de convecção, obtendo o valor 31,9243 para convecção forçada e 3,6008 para convecção natural. Sendo o maior, o primeiro valor foi o utilizado e com o mesmo foram obtidos os resultados mostrados na Tabela 3 .

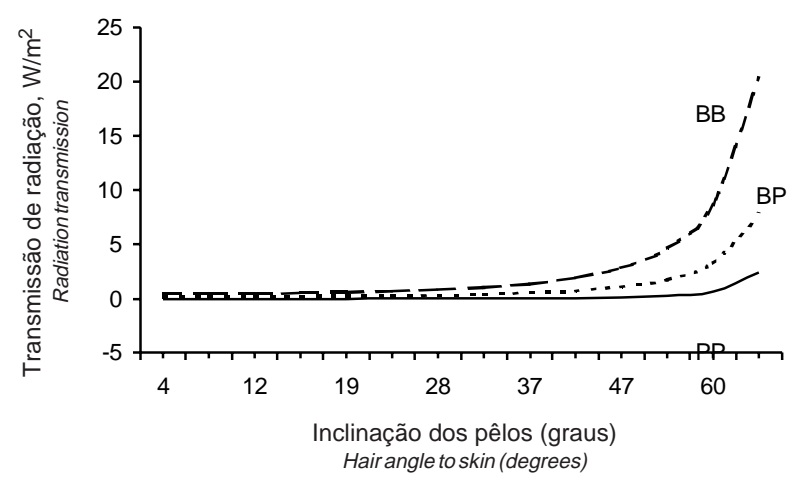

Figura 1 - Balanço térmico radiante de vacas Holandesas ao sol e à sombra, conforme a área relativa de malhas negras.

Figure 1 - Radiant heat balance of Holstein cows in sun and in shade, according to the relative area of black spots.
Tabela 3 - Temperatura radiante média $\left(T_{\mathrm{RM}}\right)$, troca térmica por ondas longas $(\mathbf{L})$ e balanço térmico por radiação $(\mathbf{R})$ em vacas Holandesas ao sol e à sombra

Table 3 - Mean radiant temperature $\left(T_{R M}\right)$, longwave thermal exchange $(\boldsymbol{L})$ and radiant heat balance of Holstein cows in sun and in shade

\begin{tabular}{lcc}
\hline $\begin{array}{l}\text { Variável } \\
\text { Variable }\end{array}$ & $\begin{array}{c}\text { Ao sol } \\
\text { Sun }\end{array}$ & $\begin{array}{c}\text { À sombra } \\
\text { Shade }\end{array}$ \\
\hline $\mathrm{T}_{\mathrm{RM}}\left({ }^{\circ} \mathrm{K}\right)$ & 356,52 & 320,49 \\
$\mathrm{~T}_{\mathrm{RM}}^{*}\left({ }^{\circ} \mathrm{K}\right)$ & 335,38 & 289,73 \\
$\mathbf{L}\left(\mathrm{W} / \mathrm{m}_{2}\right)$ & 171,84 & 99,97 \\
$\mathbf{R}\left(\mathrm{W} / \mathrm{m}_{2}\right)$ & 814,73 & 265,69 \\
\hline
\end{tabular}

Os valores de $\mathrm{R}$ dependem da proporção da área de superfície do animal coberta por pelame preto. Como se pode observar na Figura 1, quanto maior for essa proporção, maior será a quantidade total de radiação absorvida, tanto ao sol como à sombra. Em consequência, espera-se que o estresse térmico seja maior nos animais predominantemente negros, quando expostos ao sol, não havendo praticamente diferença a esse respeito quando os animais estão à sombra. Entretanto, é relativamente pequeno o incremento na radiação recebida, à medida que a área de malhas negras aumenta de 20 para $80 \%$ em relação à superfície corporal, conforme se constata na Figura 1.

Segundo os resultados de GOODWIN et al. (1995, 1997), na Austrália, vacas predominantemente brancas tenderiam a apresentar maior produção de leite que as predominantemente negras $(\mathrm{P}<0,05)$. No primeiro trabalho, as médias dos três rebanhos usados mostram que o de maior produção $(21,4 \mathrm{~kg} / \mathrm{dia} / \mathrm{vaca})$ era composto na sua maior parte por vacas predominantemente brancas, ao passo que os outros rebanhos, com produções médias menores que $19 \mathrm{~kg} / \mathrm{dia} /$ vaca, apresentaram maior número de vacas predominantemente negras. A julgar pela estrutura dos dados, é mais provável que a diferença de produção média tenha sido devida ao mérito genético médio maior no rebanho predominantemente branco. Por outro lado, vacas predominantemente negras, quando expostas ao sol, aumentaram a temperatura retal e reduziram a produção diária em maior proporção que as predominantemente brancas. No segundo trabalho, foram usadas apenas 26 vacas, das quais apenas seis apresentaram menos de $30 \%$ da área de cor negra. HANSEN (1990) apresentou resultados semelhantes, obtidos na Flórida.

Nos casos citados os autores basearam-se na 
Rev. bras. zootec.

resposta circunstancial dos animais, obtida logo após exposição à radiação solar. Esses resultados refletem apenas o fenômeno mostrado na Figura 1 agravamento do estresse térmico, devido ao maior balanço térmico radiante, não constituindo de fato uma prova completa da superioridade dos indivíduos predominantemente brancos, particularmente no tocante à radiação ultravioleta, que não foi mencionada nesses trabalhos.

BORGES (1985) observou o comportamento de 28 vacas Holandesas ao longo de 12 meses, verificando que o tempo dedicado ao pastejo sob o sol nas horas mais quentes do dia esteve positivamente associado à maior proporção de área de malhas negras. Por outro lado, FINCH et al. (1984) observaram um caso similar em gado de corte, também na Austrália, segundo o qual novilhos Shorthorn brancos pastavam mais tempo e ganhavam $0,13 \mathrm{~kg} /$ dia a mais em peso que os indivíduos vermelho-escuros.

É evidente que a questão de os animais com pelagens escuras adquirirem ou não maior carga térmica de radiação solar, com relação aos com pelagens mais claras, tem sido investigada por muitos pesquisadores com resultados contraditórios. GEBREMEDHIN et al. (1997), concordando com WALSBERG (1983), relatam que "não há uma resposta geral possível à questão ..., uma vez que tanto as considerações teóricas, como as medições empíricas,

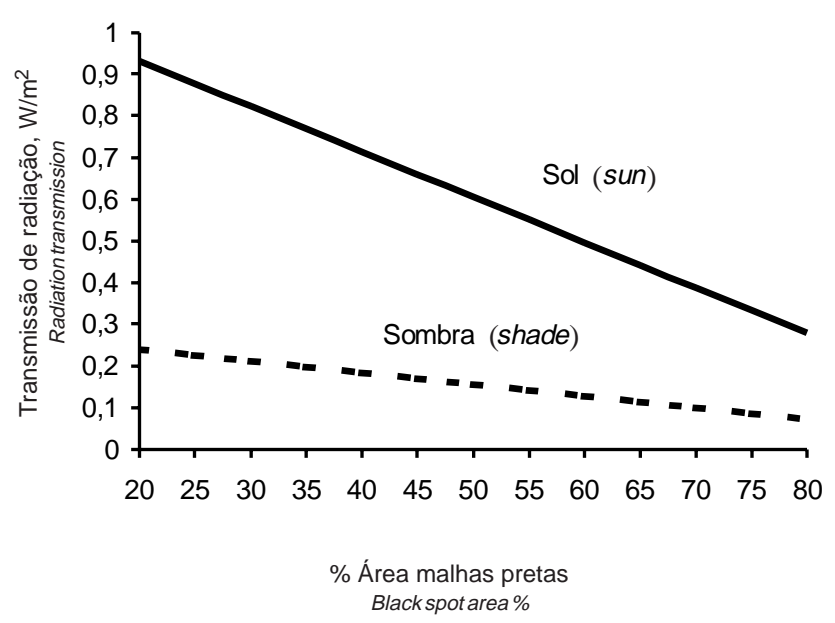

Figura 2 - Transmissão de radiação ultravioleta $(\lambda=0,2$ a 0,4 $\mu \mathrm{m})$ através do pelame e da epiderme de vacas Holandesas ao sol e à sombra, conforme a área relativa de malhas negras.

Figure 2 - Transmission of ultraviolet radiation $(\lambda=0.2$ to $0.4 \mu \mathrm{m})$ through the haircoat and the skin of Holsteins in the sun and in the shade, according to the relative area of black spots.
1409

têm demonstrado não existir relação simples entre coloração do pelame e ganho de energia radiante. Pelame escuro adquire maior ou menor quantidade de calor, dependendo de um conjunto complexo de propriedades do organismo e do ambiente, frequentemente independentes da cor do pelame."

$\mathrm{Na}$ Figura 2, pode-se observar que, se o pelame de um animal for predominantemente branco, apresentará maior penetrância à radiação ultravioleta 0,2 a $0,4 \mu \mathrm{m})$, concordando com observações de outros autores (CENA e MONTEITH, 1975; HUTCHINSON e BROWN, 1969; e WALSBERG et al., 1978). Este efeito é tanto mais acentuado quanto maior for o ângulo de inclinação dos pêlos em relação à superfície da epiderme (Figura 3). Sob irradiância de ondas curtas da ordem de 712,86 W.m ${ }^{-2}$, uma capa com pêlos brancos de $15 \mathrm{~mm}$ de comprimento médio permite que a epiderme subjacente receba e transmita $81,4 \mathrm{~W} . \mathrm{m}^{-2}$, quando os pêlos estão erectos $\left(70^{\circ} \mathrm{de}\right.$ inclinação), mas apenas $7 \mathrm{~W} . \mathrm{m}^{-2}$, quando estão mais assentados $\left(40^{\circ}\right)$. Sob esta última inclinação, os pêlos negros oferecem proteção quase total e penetração de apenas $24 \mathrm{~W} . \mathrm{m}^{-2}$, quando erectos. Deve ser recordado, no entanto, que estes resultados referemse a um caso em que a pigmentação da epiderme acompanha a do pelame, como ocorre na maioria das raças bovinas de origem européia. Na raça Jersey e naquelas nativas de regiões tropicais, a pigmentação

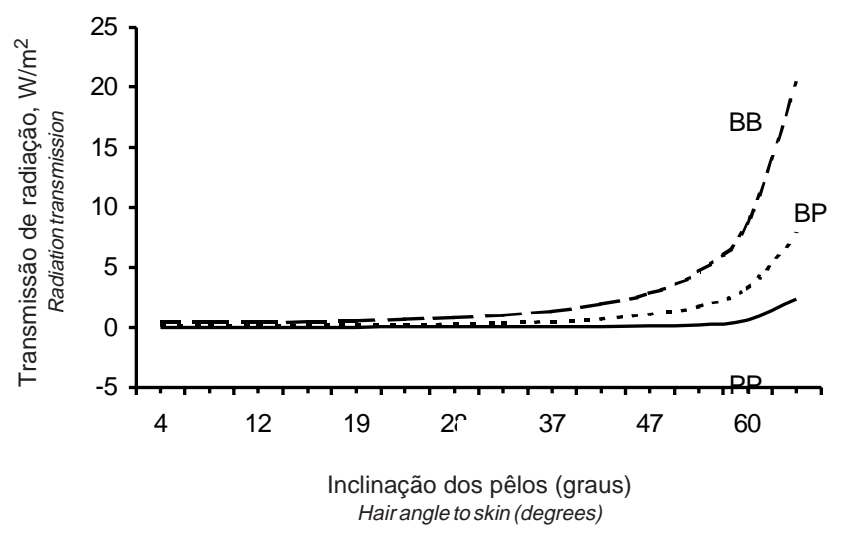

Figura 3 - Transmissão de radiação ultravioleta $(\lambda=0,2$ a 0,4 $\mu \mathrm{m})$ através do pelame e da epiderme de bovinos, de acordo com o grau de ereção dos pêlos e a pigmentação da epiderme e do pelame. Comprimento fixo dos pêlos $(15 \mu \mathrm{m})$. $\mathrm{PP}=$ pelame preto/epiderme preta, $\mathrm{BB}=$ pelame branco/ epiderme branca, $\mathrm{BP}=$ pelame branco/epiderme preta.

Figure 3 - Transmition of ultraviolet radiation ( $\lambda=0.2$ to $0.4 \mu \mathrm{m})$ through the haircoat and the skin of catle, according to the average hair angle to the skin. Fixed hair length (15 $\mu m) . P P=$ black hair/black skin, $B B=$ white hair/white skin, $B P=$ white hair/black shin. 
da epiderme não está associada à do pelame e é geralmente oposta: capa clara e epiderme escura.

A única e importantíssima vantagem da pigmentação escura da epiderme é a proteção contra a radiação ultravioleta, cuja faixa mais importante do ponto de vista biológico é entre 0,29 e $0,32 \mu \mathrm{m}$. A radiação solar nas zonas intertropicais apresenta nessa faixa energia suficiente para produzir efeitos significativos, como quebras de DNA e indução a melanomas e carcinomas epidérmicos, de modo tanto mais intenso quanto menor o comprimento de onda. Entre esses problemas, acha-se o câncer do olho, cuja incidência é elevada em regiões ensolaradas; na Austrália, a incidência atinge $2,83 \%$ nas raças com pigmentação cutânea baixa ou ausente (NISHIMURA e FRISCH 1977), ao passo que é nula nas raças altamente pigmentadas.

Observações realizadas no Laboratório de Bioclimatologia da UNESP em Jaboticabal, SP (SILVA e LaSCALA 1998, em preparação) mostraram para a epiderme de áreas pigmentadas de vacas Holandesas transmissividade de 0,098 nos comprimentos de onda entre $0,2 \mathrm{e} 0,4 \mu \mathrm{m}$, ao passo que para a epiderme não pigmentada o valor era 0,25 .

As vantagens de uma epiderme altamente pigmentada coberta com pelame branco tornam-se evidentes pela observação da Figura 3, na qual se constata que a transmissão de radiação UV através da epiderme é consideravelmente maior no caso de epiderme e pelame brancos que em outras combinações, sendo sempre menor quando a epiderme é pigmentada. Em adição, verifica-se que a proteção contra a radiação UV depende ainda da posição dos pêlos em relação à superfície da epiderme, sendo maior para pêlos assentados e com inclinação menor que $40^{\circ}$. Nestas condições, também fica mais favorecida a dissipação de energia térmica para o exterior, particularmente por evaporação.

Em consequência, o tipo mais vantajoso de bovino para regiões tropicais é aquele que apresenta uma capa de pelame branco, com pêlos bem assentados, sobre uma epiderme altamente pigmentada. Entretanto, uma vez que na raça Holandesa a pigmentação da epiderme acompanha a do pelame, temos duas alternativas: 1) se o regime de manejo for pasto, dar preferência a animais predominantemente negros, proporcionando sombra suficiente na pastagem; 2) se o regime for de estabulação, animais predominantemente brancos serão mais vantajosos; em qualquer caso, a capa de pelame deverá ser a menos espessa possível, com pêlos curtos, grossos e bem assentados.

\section{Conclusões}

Animais predominantemente negros são melhor protegidos contra a radiação de ondas curtas que os predominantemente brancos, particularmente aqueles com capa de pelame mais finas (4 a $7 \mathrm{~mm}$ ), os quais são mais adequados a um ambiente tropical.

\section{Agradecimento}

Ao Dr. John E. Frisch, pelo acesso praticamente irrestrito à toda a documentação da biblioteca e dos arquivos do Tropical Beef Centre, Rockhampton, Queensland, Australia, onde foi realizada parte da pesquisa bibliográfica.

\section{Referências Bibliográficas}

ALMEIDA, A.G.C. Comprimento dos pêlos, ângulo de inclinação dos pêlos, espessura de pelame e a produção de leite de vacas da raça Holandesa. Jaboticabal: FCAVJ-UNESP, 1986. Monografia de graduação - Faculdade de Ciências Agrárias e Veterinárias/ UNESP, 1986.

BAKKEN, G.S. 1976. A heat transfer analysis of animals: unifying concepts and the application of metabolism chamber data to field ecology. J. Theoret. Biol., 60:337-340.

BERMAN, A., VOLCANI, R. 1961. Seasonal and regional variations in coat characteristics of dairy cattle. Aust. J. Agric. Res., 12:528538.

BIANCA, W., WEGMANN-BOSSHARDT, H., NÄF, F. 1974. Untersuchungen an Rinderhaaren. Z. Tierzüchtg. Züchtgsbiol., 9:217-226.

BLAXTER, K. 1989.Energymetabolisminanimalsandman. Cambridge: Cambridge Univ. Press. 336p.

BORGES, F.M.A. Relações entre as características do pelame, a taxa de sudação e o comportamento de vacas Holandesas na pastagem. Jaboticabal: FCAVJ/UNESP, 1985. Monografia de GraduaçãoFaculdade de Ciências Agrárias e Veterinárias/UNESP, 1985.

CAMPBELL, G.S. 1977. An Introduction to environmentalbiophysics. New York: Springer-Verlag. 285p.

CENA, K. 1966. Observations on the absorption of solar radiation in cows and horses of various colors. Acta Agric. Scand. 6:93-138.

CENA, K., MONTEITH, J.L. 1975. Transfer processes in animal coats. I. Radiative transfer. Proc. Royal Soc., 188:377-394.

CHAPMAN, A.J. 1987. Fundamentals of heat transfer. New York: McMillan. 751p.

CLAPPERTON, J.L., JOYCE, J.P., BLAXTER, K.L. 1965. Estimates of the contribution of solar radiation to thermal exchanges of sheep. J. Agric. Sci., 64:37-49.

COWLES, R.B. 1967. Black pigmentation: adaptation for concealment or heat conservation? Sci., 158:1340-1341.

DAWSON, T.J., BROWN, G.D. 1970. A comparison of the insulative and reflective properties of the fur of desert kangaroos. Comp. Biochem. Physiol., 37:23-38.

ESMAY, M.L. 1969. Principles of animal environment. Westport, Con.: AVI Publ. Co.

FINCH, V.A., BENNETT, I.L., HOLMES, C.R. 1984. Coat colour in cattle: effect on thermal balance, behaviour and growth, and relationship with coat type. J. Agric. Sci. 102:141-147.

GEBREMEDHIN, K.G., NI, H., HILLMAN, P.E. Temperature 
Rev. bras. zootec.

profile and heat flux through irradiated fur layer. In: USA INTERNATIONAL LIVESTOCK ENVIRONMENT SYMPOSIUM, 5, 1997, Bloomington, MN. Proceedings... 1. St. Joseph, MI: American Society of Agricultural Engineers, 1997, p.226-241.

GOODWIN, P., JOSEY, M.J., COWAN, J.M. Coat color and its effect on production in Holstein-Friesians in South-East Queensland. In: AUSTRALIA - AUSTRALIAN ASSOCIATION OF ANIMAL BREEDING AND GENETICS CONFERENCE, 11, 1995. Proceedings... p. 295-298, 1995.

GOODWIN, P., GAUGHAN, J., SKELE, P. et al. Coat color and alleviation of heat load in Holstein-Friesian cows. In: USA INTERNATIONAL LIVESTOCK ENVIRONMENT SYMPOSIUM, 5, 1997, Bloomington, MN. Proceedings... 2. St. Joseph, MI: American Society of Agricultural Engineers, p.923927, 1997.

HAMILTON, W.J. 1973. Life's color code. New York: McGrawHill.

HAMILTON, W.J., HEPPNER, F. 1967. Radiant solar energy and the function of black homeotherm pigmentation: an hypothesis. Sci., 155:196-197.

HANSEN, P.J. Effects of coat colour on physiological responses to solar radiation in Holstein. Veterinary Record, 127:333-334, 1990.

HUTCHINSON, J.C.D., BROWN, G.B. 1969. Penetrance of cattle coats by radiation. J. Applied Physiol., 26:454-464.

KOVARIK, M. 1964. Flow of heat in an irradiated protective cover. Nature, 201:1085-1087.

KOVARIK, M. 1973. Radiation penetrance of protective covers. $J$. Applied Physiol., 35:562-563.

McARTHUR, A.J. 1987. Thermal interaction between animal and microclimate: acomprehensivemodel.J.Theoret.Biol., 126:203-238.

McCULLOUGH, E.M., PORTER, W.P. 1971. Computing clear day solar radiation spectra for the terrestrial ecological environment. Ecology, 52:1008-1015.

MONTEITH, J.L. 1974. Specification of the environment for thermal physiology. In: MONTEITH, J.L., MOUNT, L.E. (Eds.) Heat loss from animals and man. London: Butterworths. p.1-17.

MONTEITH, J.L., UNSWORTH, M.H. 1991. Principles of environmental physics. 2.ed. London. 291p.

MOUNT, L.E. 1968. The climatic physiology of the pig. London: Ed. Arnold. 300p.
NISHIMURA, H., FRISCH, J.E. Eye cancer and circumocular pigmentation in Bos taurus, Bos indicus and crossbred cattle. Aust. J. Exper. Agric. Anim. Husb. 17:709-711, 1977.

PEGLER JR., F. Proporção da área de malhas escuras em relação à de malhas brancas x produção de leite. Jaboticabal: FCAVJUNESP, 1986. 40p. Monografia (Graduação em Zootecnia) Universidade Estadual Paulista, 1986.

PINHEIRO, M.G. Variação genética de características da capa externa de vacas da raça Holandesa em ambiente tropical. Ribeirão Preto: USP, 1996. Tese (Doutorado em Genética), Universidade de São Paulo, 1996.

STAFFORD-SMITH, D.M., NOBLE, I.R., JONES, G.K. 1985. A heat balance model for sheep and its uses to predict shade-seeking behaviour in hot conditions. J. Applied Ecol., 22:753-774.

SILVA, R.G., ARANTES NETO, J.G., HOLTZ FILHO, S.V. 1988. Genetic aspects of the variation of the sweating rate and coat characteristics of Jersey cattle. Braz. J. Gen., 11:335-347.

SILVA, R.G., LaSCALA JR., N. 1998. Transmissão de ondas curtas através da epiderme de bovinos. Trabalho em preparação.

STEWART, R.E. 1953. Absorption of solar radiation by the hair of cattle. Agric. Engineering, 34:235-238.

UDO, H.M.J. 1978. Hair coat characteristics in Friesian heifers in the Netherlands and Kenya. Meded.Landbouwhogeschool Wageningen, 78-6.

WALSBERG, G.E. 1983. Coat color and solar energy gain in animals. BioScience, 33:88-91.

WALSBERG, G.E., CAMPBELL, G.S., KING, J.R. 1978. Animal coat color and radiative heat gain: are-evaluation. J.Comp.Physiol., 126:211-222.

Recebido em: 04/12/98

Aceito em: 12/04/99 